\title{
STRETCHING PHENOMENA IN MAPPINGS OF SPHERES ${ }^{1}$
}

H. BLAINE LAWSON, JR.

This paper presents an extension of the recent work of R. Olivier [3] on dilatation phenomena in differentiable mappings of spheres. Let $S^{k}=\left\{\left(x_{1}, \cdots, x_{k+1}\right) \in R^{k+1}: \sum_{j=1}^{k+1} x_{j}^{2}=1\right\}$ be provided with the usual unit sphere metric $d$. Let $f: S^{m} \rightarrow S^{n}$ be a differentiable mapping and define a dilatation constant $\delta_{f}=\max \left\|f_{*}(X)\right\| /\|X\|$, where $X$ runs over the nonzero tangent vectors of $S^{m}$ and where $f_{*}$ is the induced mapping on the tangent vectors. Olivier showed that if $m=n$ and $f$ has even nonzero degree, or if $m>n=2$ and $f$ is not homotopic to zero (designated $f \not 20$ ), then $\delta_{f} \geqq 2$.

We prove a generalization conjectured by Olivier.

THEOREM 1. If $m>n$ for any $n>0$ and if $f \not x 0$, then $\delta_{f} \geqq 2$.

Proof. The Borsuk-Ulam theorem [3, p. 266] guarantees the existence of a point $x \in S^{m}$ such that $f(x)=f(-x)$. Assume $\delta_{f}<2$. Then each meridian in $S^{m}$ from $-x$ to $x$ is mapped into a loop at $a=f(x)$ of length less than $2 \pi$. Hence $-a$ does not belong to the image $f\left(S^{m}\right)$ and therefore $f \simeq 0$.

Let $\{f\}$ denote the homotopy class of $f$ in $\pi_{m}\left(S^{n}\right)$ and let $\Sigma: \pi_{m-1}\left(S^{n-1}\right) \rightarrow \pi_{m}\left(S^{n}\right)$ be the suspension homomorphism.

ThEOREM 2. Let $m=2 k>0$ and assume that $2\{f\} \neq 0$. Then if $\{f\} \notin \Sigma\left(\pi_{2 k-1}\left(S^{n-1}\right)\right), \delta_{f} \geqq 3$.

This statement is of interest only for $k \geqq n$ but has the following interesting corollary.

CoRollary 1. If $f: S^{2 k} \rightarrow S^{2}$ is a differentiable mapping with $k>2$ and $2\{f\} \neq 0$, then $\delta_{f} \geqq 3$.

We first prove a

LEM MA. Let $f: S^{2 k} \rightarrow S^{n}$ with $k>0$ b e any mapping such that $2\{f\} \neq 0$ Then there exists a point $x \in S^{2 k}$ such that $f(x)=-f(-x)$.

Proof. Suppose that no such point exists. Then the mapping $\phi: S^{2 k} \rightarrow S^{n}$, where $\phi(x)=(f(x)-f(-x)) /|f(x)-f(-x)|$ is well defined. (The operations are done in $R^{n+1}$.) Clearly, $\phi \simeq f$ and $\phi \cdot A=\phi$,

Received by the editors January 14, 1967.

1 This research was supported in part by NFS Grant GP-6977. 
where $A: S^{2 k} \rightarrow S^{2 k}$ is the antipodal map. Hence the following triangle commutes.

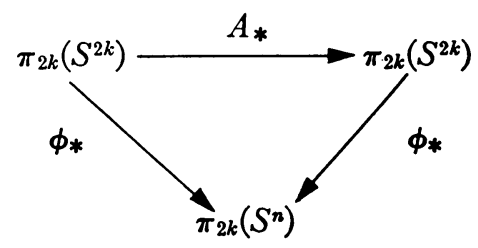

Setting $1=\{$ identity $\} \in \pi_{2 k}\left(S^{2 k}\right)$, we obtain $\{f\}=\phi_{*}(1)=\phi_{*}\left(A_{*}(1)\right)$ $=\phi_{*}(-1)=-\{f\}$ and $2\{f\}=0$ contrary to assumption.

Proof of Theorem 2. Suppose that $2\{f\} \neq 0$ but that $\delta_{f}<3$. By the Freudenthal theorems and Theorem 1, it suffices to consider $k \geqq n$ and $\delta_{f} \geqq 2$. Let $\lambda$ be a number such that $0<\lambda<\pi$ and $\delta_{f}<2+\lambda / \pi$. By the lemma there is a point $x$ in $S^{m}$ such that $d(f(x), f(-x))=\pi$; and by Borsuk-Ulam [3, p. 266], there is a point $x^{\prime}$ such that $d\left(f\left(x^{\prime}\right), f\left(-x^{\prime}\right)\right)$ $=0$. Thus by the continuity of $d$ there is a point $y \in S^{m}$ such that $d(f(y), f(-y))=\lambda$.

Denote by $\Omega^{c} S^{l}(a, b)$ the set of all piecewise smooth paths $\gamma:[0,1] \rightarrow S^{l}$ such that $\gamma(0)=a, \gamma(1)=b$ and $\int_{0}^{1}\|d \gamma / d t\|^{2} d t \leqq c^{2}$, with the compact-open topology. Observe that if $\gamma \in \Omega^{c} S^{l}(a, b)$, then the length of $\gamma$ is less than or equal to $c$ due to the Schwarz inequality.

By definition of $\lambda, \delta_{f} \pi<2 \pi+\lambda$. Therefore, since $f(y)$ and $f(-y)$ are not conjugate along any geodesic in $S^{n}$, a standard application of Morse Theory [2, p. 96] shows that

$$
\Omega^{\delta f} \stackrel{\text { def. }}{=} \Omega^{\delta f \pi} S^{n}(f(y), f(-y))
$$

has the homotopy type of a $C$ - $W$ complex with one cell in dimension zero and one cell in dimension $n-1$, i.e., $\Omega^{\delta_{f}}$ has the homotopy type of $S^{n-1}$. Observe that in a natural way $f$ induces a mapping $\tilde{f}: \Omega^{\pi} S^{2 k}(y,-y) \rightarrow \Omega^{\delta}$. Clearly, $\Omega^{\pi} S^{2 k}(y,-y)$ is homeomorphic to $S^{2 k-1}$ and $\tilde{f}$ determines a unique class in $\pi_{2 k-1}\left(S^{n-1}\right)$.

It remains only to show that $\Sigma(\{\tilde{f}\})=\{f\}$. Let

$$
\Omega S^{n}=\Omega S^{n}(f(y), f(-y))
$$

be the full path space of $S^{n}$ and $i: \Omega^{\delta f} \subset \Omega S^{n}$ the inclusion map. Denote by

$$
\theta: \quad \pi_{2 k}\left(S^{n}\right) \underset{\rightarrow}{\rightarrow} \pi_{2 k-1}\left(\Omega S^{n}\right)
$$

the standard adjoint isomorphism. Then the class in $\pi_{n-1}\left(\Omega S^{n}\right)$ deter- 
mined by $i$ corresponds to the adjoint of the identity map of $S^{n}$, and the following diagram commutes.

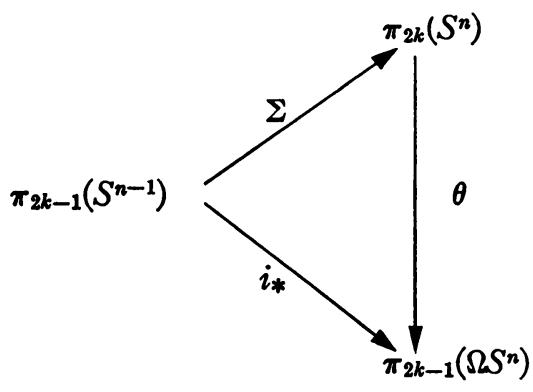

From the construction it is clear that $i_{*}(\{\bar{f}\})=\theta(\{f\})$, and thus $\Sigma(\{\tilde{f}\})=\{f\}$. This completes the proof.

I would like to thank Professor Hans Samelson for several suggestions helpful in developing these ideas.

\section{BibliogRAPHY}

1. John Milnor, Morse theory, Princeton Univ. Press, Princeton, N. J., 1963.

2. Reinhard Olivier, Über die Dehnung von Sphärenabbildungen, Inventiones Math. 1 (1966), 380-390.

3. Edwin Spanier, Algebraic topology, McGraw-Hill, New York, 1966.

STANFORD UNIVERSITY 